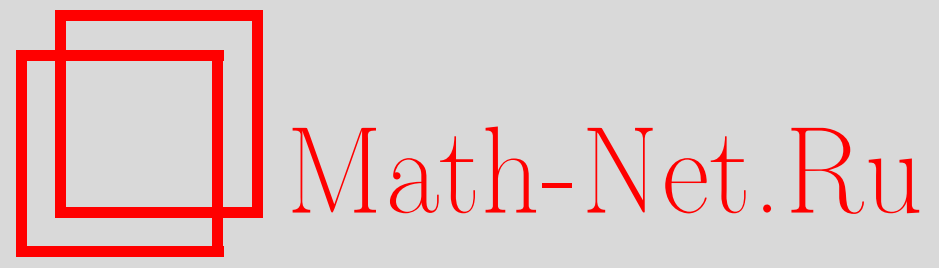

Ю. В. Туровский, В. С. Шульман, Инвариантные подпространства операторных алгебр Ли и теория $K$-алгебр, Функи. анализ и его прил., 2002, том 36, выпуск 4, 88-91

DOI: https://doi.org/10.4213/faa226

Использование Общероссийского математического портала MathNet.Ru подразумевает, что вы прочитали и согласны с пользовательским соглашением

http://www. mathnet.ru/rus/agreement

Параметры загрузки:

IP : 54.197 .130 .99

26 апреля 2023 г., 17:09:40

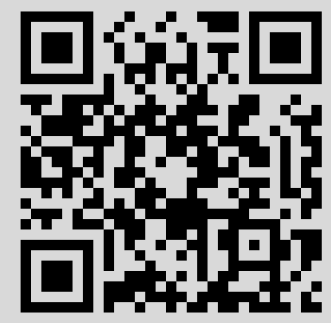


УДК 517.983

\title{
Инвариантные подпространства операторных алгебр Ли и теория $K$-алгебр
}

\author{
(c) 2002. Ю. В. ТуровсКий, В. С. ШульмАн
}

Данная работа находится на стыке теорий бесконечномерных алгебр Ли и инвариантных подпространств. Изучение таких алгебр, подчиненных некоторым условиям компактности, проводится с помощью анализа их неприводимых представлений, который, в свою очередь, основывается на существовании общего инвариантного подпространства у семейства компактных операторов, связанных лиевыми соотношениями. Фундаментом этого направления являются теоремы Энгеля и Ли, утверждающие, что неприводимые конечномерные представления нильпотентных и соответственно разрешимых конечномерных комплексных алгебр Ли одномерны. Если рассматривать бесконечномерные представления компактными операторами, то доказать аналогичные теоремы далеко не просто даже в случае коммутативных алгебр Ли; для одномерных алгебр это было сделано фон Нейманом, Ароншайном и Смитом [1], для алгебр произвольной размерности - В. И. Ломоносовым [2].

Естественный банахов аналог класса нильпотентных (соответственно конечномерных) алгебр Ли - энгелевы алгебры Ли (соответственно $K$-алгебры Ли), т.е. такие алгебры Ли, на которых операторы присоединенного представления квазинильпотентны (соответственно компактны). «Компактная» версия теоремы Энгеля получена в [3]: неприводимые представления энгелевой алгебры Ли компактными операторами одномерны. Для классически разрешимых (т. е. тех, у которых производный ряд обрывается на конечном шаге) бесконечномерных алгебр Ли соответствующий «компактный» аналог получен в [4].

Мы установим одномерность неприводимых представлений более широкого класса, естественного аналога класса разрешимых в категории банаховых алгебр Ли.

ОПрЕДЕЛЕНИЕ. Банахова алгебра Ли $L$ называется $E$-разрешимой или алгеброй Ли, разрешимой по Энгелю (соответственно HK-алгеброй Ли или, подробнее, алгеброй Ли с гипокомпактным присоединенным действием), если для любого замкнутого собственного идеала $J$ факторалгебра $L / J$ имеет ненулевой замкнутый энгелев идеал (соответственно идеал, являющийся $K$-алгеброй).

Полученные результаты проясняют структуру банаховых алгебр Ли с компактным присоединенным действием (т.е. $K$-алгебр Ли), исследования которых были начаты в семидесятые годы Гурарием и Ваксманом [5] и Войтыньским $[6,7]$.

1. Пусть, как обычно, $B(X)$ - алгебра всех ограниченных линейных операторов в банаховом пространстве $X, K(X)$ - идеал всех компактных операторов, а структура алгебры Ли в $B(X)$ задана коммутатором $[T, S]=T S-S T$. Oneраторная алгебра Ли - это подалгебра алгебры Ли $B(X)$. Если $M \subset B(X)$, то через $A(M)$ обозначается замкнутая (ассоциативная) подалгебра в $B(X)$, порожденная $M$. Замкнутое подпространство $Y \subset X$ называется инвариантным для множества операторов $M \subset B(X)$, если $T Y \subset Y$ при любом $T \in M ; Y$ гиперинвариантно для $M$, если оно инвариантно для $M \cup M^{\prime}$, где $M^{\prime}=\{S \in$ 
$B(X):[S, T]=0, T \in M\}-$ коммутант множества $M$. Множество операторов называется вольтерровым, если его элементы вольтерровы, т. е. компактны и квазинильпотентны.

Начнем с вопросов существования инвариантных подпространств у алгебр Ли компактных операторов. Наш первый результат устанавливает, что наличие (обилие) в операторной алгебре Ли операторов конечного ранга является условием, препятствующим существованию инвариантных подпространств, что для теории инвариантных подпространств несколько необычно.

Теорема 1.1. Если замкнутая алгебра Ли компактных операторов имеет ненулевой идеал, не содержащий ненулевых операторов конечного ранга, то она имеет нетривиальное инвариантное подпространство.

Следующая теорема - центральный результат статьи; на ней основаны доказательства большинства последующих утверждений.

Теорема 1.2. Если алгебра Ли L компактных операторов имеет ненулевой вольтерров идеал $J$, то у нее есть нетривиальное гиперинвариантное подпространство.

Частный случай теоремы 1.2 - это теорема о существовании инвариантных подпространств для вольтерровых алгебр Ли [3]. Разумеется, доказательство теоремы 1.2 (так же, как и теоремы 1.1) использует этот результат, но вместе с тем требует достаточно кропотливого анализа спектральных многообразий операторов дифференцирования вольтерровых алгебр. Метод работает и при более слабых ограничениях. Так, теорема 1.2 останется верной, если предполагать, что $L$ состоит из операторов Рисса (но, правда, в таком случае $L$ должна быть замкнутой), а $J$ можно считать $E$-разрешимым. Если же $J$ порожден операторами конечного ранга (для $L \subset K(X)$ это основной случай, как показывает теорема 1.1), то от $L$ можно лишь требовать, чтобы она состояла из операторов со счетными спектрами.

СледствиЕ 1.3. Пусть L - операторная алгебра Ли. Среди всех вольтерровых идеалов алгебры $L$ есть наибольший идеал $V(L)$; он состоит из всех операторов $T \in L$, которые принадлежат радикалу Джекобсона алгебры $A(L \cap K(X))$.

Скажем, что оператор $T \in B(X)$ дифференцирует множество операторов $M$, если $[T, S] \in M$ для всех $S \in M$.

СледствиЕ 1.4. Пусть $A-$ алгебра вольтерровых операторов в X. Тогда существует нетривиальное подпространство $Y \subset X$, инвариантное для всех компактных операторов, дифференцирующих $A$.

Неизвестно, можно ли здесь снять ограничение компактности дифференцирующих операторов. В некоторых важных случаях ответ утвердителен.

СледствиЕ 1.5. Пусть А-коммутативная алгебра вольтерровых операторов, замыкание которой в слабой операторной топологии содержит единичный оператор (или, более общим образом, такая, что пересечение замьканий в слабой операторной топологии алгебр $A^{n}$ не сводится к нулю; здесь $A^{n}$ - алгебра, порожденная всеми произведениями $n$ элементов алгебрь $\left.A\right)$. Тогда существует нетривиальное подпространство, инвариантное для всех операторов, дифференцирующих $A$. 
Ситуацию хорошо иллюстрирует пример алгебры, порожденной вольтерровым оператором интегрирования $V$. Существует много операторов, которые ее дифференцируют, однако все они сохраняют (исследованную во многих работах) решетку инвариантных подпространств оператора $V$.

ТеоремА 1.6. Пусть L-конечномерная алгебра Ли компактных операторов в бесконечномерном банаховом пространстве. Тогда существует нетривиальное подпространство, инвариантное для всех операторов, дифференцирующих $L$.

2. Пусть $L$ - банахова алгебра Ли, т. е. алгебра Ли, являющаяся банаховым пространством со скобкой Ли, совместно непрерывной по обоим аргументам. Ее представлением называется непрерывный (лиев) гомоморфизм в $B(X)$. В частности, присоединенное представление $\operatorname{ad}_{L}$ действует на самой $L$ по формуле $\left(\operatorname{ad}_{L} a\right)(x)=[a, x]$.

ТЕОРЕма 2.1. Всякое неприводимое представление Е-разрешимой банаховой алгебры Ли компактными операторами одномерно.

Теорема 2.2. Всякое неприводимое представление НК-алгебры Ли компактными операторами конечномерно.

СледствиЕ 2.3. Всякий минимальный замкнутый лиев идеал $K$-алгебры Ли конечномерен (и, значит, либо коммутативен, либо полупрост).

В работе [5] был введен класс разрешимых $K$-алгебр Ли. Такие $K$-алгебры Ли определялись двумя эквивалентными условиями: отсутствием конечномерных полупростых подалгебр или факторалгебр Ли. Там же было доказано, что всякая $K$-алгебра Ли $L$ содержит наибольший разрешимый идеал $R(L)$.

ТеоремА 2.4. (а) K-алгебра Ли разрешима в смысле [5] тогда и только тогда, когда она Е-разрешима.

(b) $K$-алгебра Ли Е-разрешима тогда и только тогда, когда все ее неприводимые представления компактными операторами одномерны.

(с) $R(L)$ образован всеми элементами $K$-алгебры Ли $L$, которым при неприводимых (конечномерных) представлениях компактными операторами соответствуют операторь, кратные единичному.

Из следствия 1.3 легко вытекает существование в любой $K$-алгебре Ли $L$ наибольшего энгелева идеала $E(L)$. Обозначим, следуя [5], через $\Lambda(L)$ пересечение ядер всех (конечномерных) неприводимых представлений компактными операторами алгебры Ли $L$.

СлеДСТВИе 2.5. $[L, R(L)] \subset \Lambda(L) \subset E(L) \subset R(L)$ для всякой $K$-алгебры Лu $L$.

В [5] эти включения и существование идеала $E(L)$ были доказаны для произвольной локально конечной $K$-алгебры Ли $L$ (т. е. имеющей плотный идеал, каждое конечное подмножество которого порождает конечномерную подалгебру Ли).

Авторы признательны Д. Белтице, М. Шабаку и В. Л. Островскому за полезные обсуждения рассматриваемых в работе вопросов.

\section{ЛитерАтУРА}

1. Aronszajn N. A., Smith K. T. Ann. Math., 60, 345-350 (1954). 2. Ломоносов В. И. Функц. анализ и его прил., 7, вып. 3, 55-57 (1973). 3. Shulman V. S., Turovskii Yu. V. 
J. Funct. Anal., 177, 383-441 (2000). 4. Shulman V. S., Turovskii Yu. V. In: Spectral and Evolutionary Problems, Vol. 9. Simferopol, 1999, pp. 38-44. 5. Ваксман Л. Л., Гурарuй Д. Л. Теория функций, функц. анализ и его прил., 24, 16-32 (1975). 6. Wojtynski W. Stud. Math., 59, No. 3, 263-273 (1977). 7. Wojtynski W. Bull. Acad. Polon. Sci., Ser. Math., 26, No. 2, 105-107 (1978).

Институт математики и механики НАН Азербайджана e-mail: yuri@sinam.net

Поступило в редакцию

Вологодский политехнический институт, кафедра математики

7 мая 2002 г.

e-mail: shulman_v@yahoo.com 